\title{
11. THE SPAGE DISTRIBUTION OF MIDDLE- AND LATE-TYPE SUPER-GIANTS IN THE REGION OF THE GALACTIC SYSTEM NEAR THE SUN *
}

\author{
WILLIAM P. BIDELMAN
}

Lick Observatory, University of California, U.S.A.

This paper is concerned with the space distribution in our own Galaxy of the most luminous stars of spectral types $\mathrm{A}_{5}$ and later which have been classified on the basis of low-dispersion slit spectrograms. Seventy stars of luminosity classes Ia and Iab on the Morgan-Keenan system [1] are considered, of which the majority have been previously noted as of high luminosity at the Yerkes, Harvard, Mount Wilson, and Warner and Swasey Observatories. The Cepheid variables have not been considered.

In order to derive the distances of these stars, one needs to know their spectroscopically assigned absolute magnitudes, which are quite uncertain, and also the interstellar absorption suffered by their light. The interstellar absorptions have been estimated in each individual case from the color excesses of neighboring early-type stars of, if possible, similar distances, using the data published recently by W.W. Morgan and his collaborators [2]. These estimated absorptions are of course subject to considerable errors, which may be especially large in the case of the southern stars. The absolute magnitude calibration used is a modification of that given by Keenan and Morgan in the book Astrophysics [3]. This modification is suggested by the undoubted presence of a considerable number of these very high luminosity stars in various stellar associations and clusters, especially in those which do not give evidence of being extremely young [4]. As an exexample of this modification, it may be noted that the most luminous stars of types near Go, such as $\rho$ Cassiopeiae and HR 4337, have been assigned visual absolute magnitude -8.5 . In view of the method of calibration used, the writer's distance scale is tied directly to that used by Morgan, Whitford and Code in their discussion of the space distribution of the blue giants [5].

The positions of the individual middle- and late-type super-giant stars,

* Contributions from the Lick Observatory, Ser. Ir, no. 8r. 


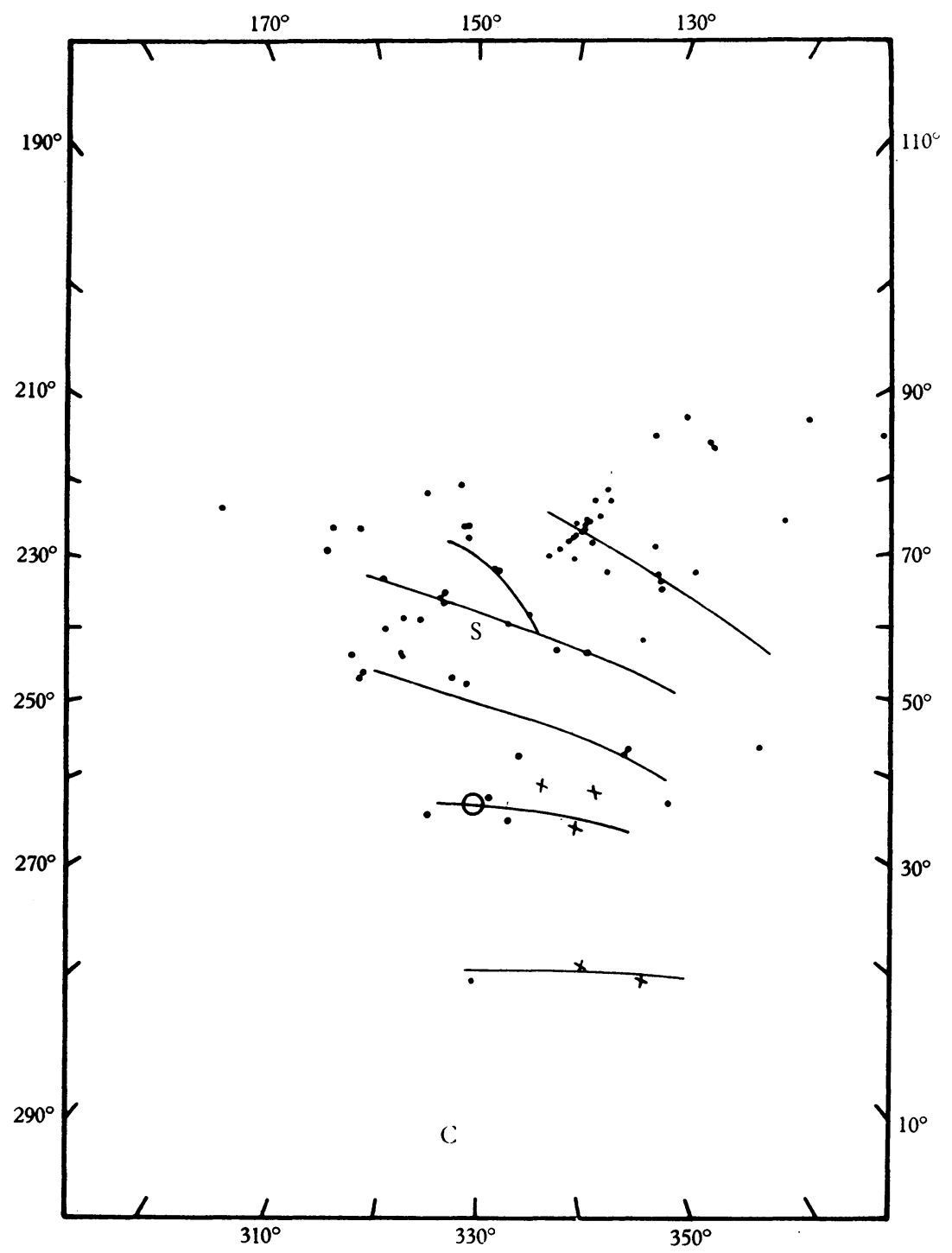

Fig. 1. The positions, projected on the galactic plane, of seventy middle- and late-type stars of high luminosity. Filled circles represent individual super-giant stars, crosses individual earlytype stars found by Morgan, Whitford and Code $(A p . J .118,318,1953)$, and the large open circle an early-type stellar association discovered by Hiltner $(A p . J .120,4 \mathrm{I}, 1954)$. The letter $\mathrm{S}$ indicates the position of the sun, $\mathrm{C}$ that of the galactic center. The curves nearest the solar position have been drawn in the positions of nearby spiral arms indicated by optical and $21-\mathrm{cm}$ radio data. 
projected on the galactic plane, are shown in Fig. I. The significance of the various symbols used is noted in the figure legend. The curves passing nearest the sun are drawn in the positions of the Perseus arm, the Orion arm, and the first inner arm, which have been previously indicated by optical and $2 \mathrm{I}-\mathrm{cm}$ radio data. The two curves nearer the galactic center have been drawn in to indicate other possible, more distant arms.

In connection with Fig. I, it must be realized that the data plotted refer to individual stars whose assigned distances probably have uncertainties of the order of $30 \%$. For example, the rather large spread in distance shown by the group of stars near the Perseus arm at galactic longitudes near $105^{\circ}$ is undoubtedly largely due to observational error, since it is quite certain that most of these stars are associated with the $h$ and $\chi$ Persei clusters, and as such should all actually be plotted at the same distance. Because of the large uncertainties in distance, and also because of the relatively small number of individual stars plotted, it is not to be anticipated that the points in Fig. I should actually define the complex spiral arms which really exist, even if these high luminosity stars do lie in fact in or near these arms. However, one can legitimately consider the question of whether these middle- and late-type super-giant stars do follow the presently accepted spiral arm pattern which has been previously and independently established. With due regard to the limitations of the data, it appears to the writer that this question can be answered, in general, in the affirmative. At the same time it should be emphasized that certain of these super-giants, 89 Herculis, for example, are located at rather large distances from the galactic plane, and hence cannot be said to be closely associated with any spiral arm of our Galaxy.

Additional radial velocities and three-color observations of these high luminosity stars will undoubtedly permit a considerably more definitive study of the space distribution of these objects.

\section{REFERENCES}

[1] Johnson, H. L. and Morgan, W. W. Ap. J. 117, 3 1 3, 1953.

[2] Morgan, W. W., Gode, A. D. and Whitford, A. E. Astrophysical Supplements, 2, 41, r 955 (no. 14).

[3] Hynek, J. A. (editor). New York (1951).

[4] Bidelman, W. P. A.J. 59, 315, 1954.

[5] Morgan, W. W., Whitford, A. E. and Code, A. D. Ap. J. 118, $3^{18,} 1953$. 